\title{
Analytical-method for polishing-surface prediction considering tool flexibility and grain-material interaction
}

\author{
Amaia LEGIDO PÉREZ DE SAN ROMÁN a,b, Julien CHAVES-JACOB a , Jean- \\ 5 Marc LINARES ${ }^{\text {a }}$, Pedro José ARRAZOLA ${ }^{\text {b }}$ \\ ${ }^{a}$ Aix Marseille Univ, CNRS, ISM, Inst Movement Sci, Marseille, France \\ b Escuela Politécnica Superior de Mondragón Unibertsitatea, Loramendi 4, 20500 \\ 10 \\ Mondragón, Spain
}

\begin{abstract}
To improve surface roughness of components without altering geometrical dimensions, polishing operations are commonly used. These operations may be timeconsuming and expensive. Thus, the prediction of polished surface quality is a key issue

15 to reduce the cost of these operations. Resulting surface quality of polishing operations is the outcome of a large number of local cutting phenomena (grains-material). The control of the multi-scale physical phenomena is a challenge when it comes to simulate these operations. In this paper, an Analytical-Method for Polishing-Surface Prediction (AMPSP) that considers the tool flexibility and each grain-material interaction is proposed. The objective is to predict accurately the polished surface topography and the material removal rate, and to keep the history of all the local cutting phenomena, in order to define a digital twin of polishing operation. Experimental validation demonstrates that this AMPSP predicts the material removal rate (less than $35 \%$ of error) and the surface topology (less than a few percent). AMPSP will enable engineers to quickly and accurately predict the polished topology obtained with 5-axis toolpaths.
\end{abstract}

Keywords: Finishing; Polishing; Modelling; Digital Twin. 


\section{List of symbols and units}

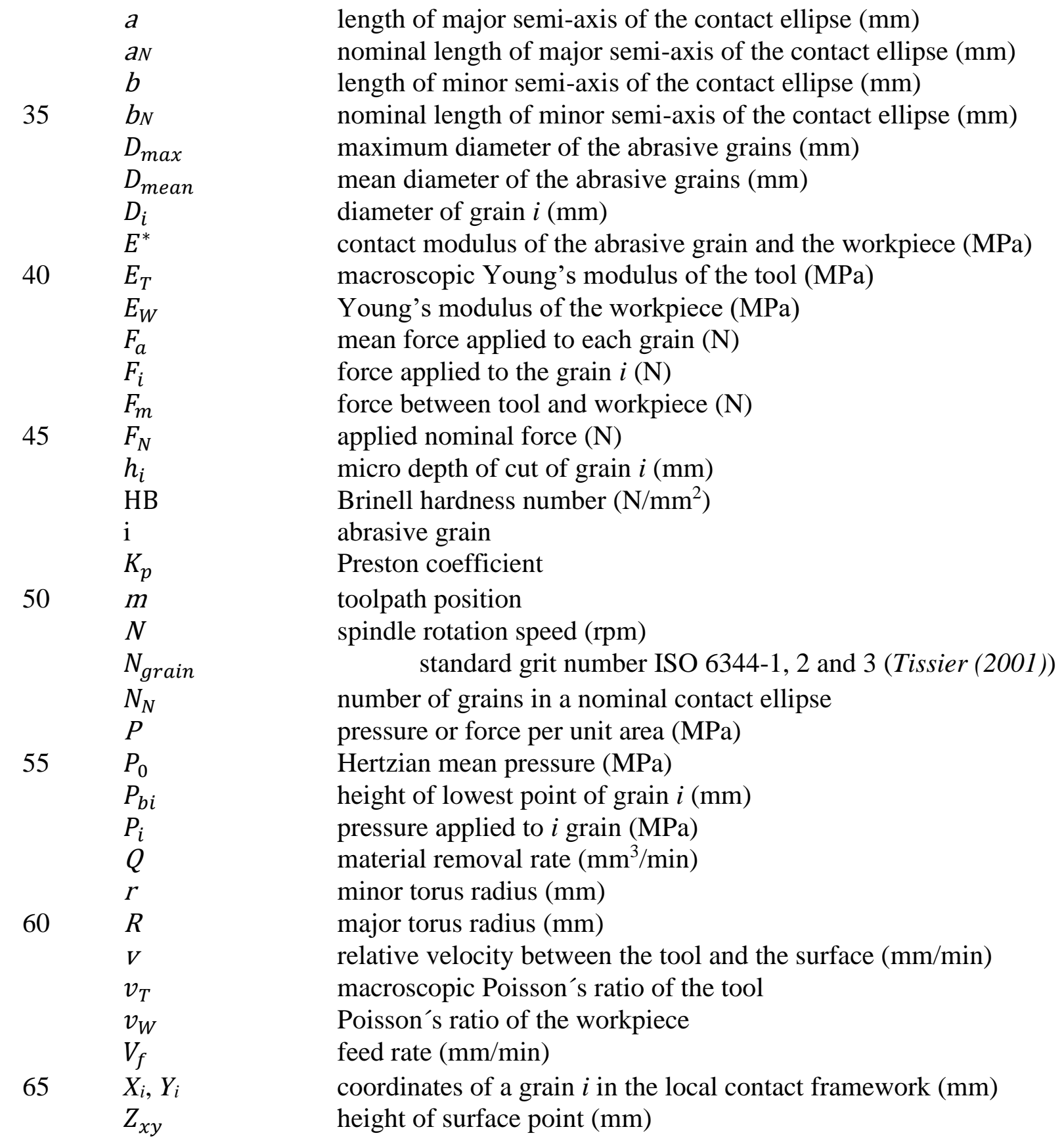

\section{Greek letters}

70

$\Delta \omega$

$\theta_{1}, \theta_{2}$

$\Delta t$

$\sigma$

75
$\omega$
$\delta_{N}$
$\delta_{i}$
$\delta_{\text {imax }}$

spindle rotation angle discretization $\left(^{\circ}\right)$ tilt and lead angles $\left(^{\circ}\right)$

discretised time (s)

standard deviation of grain diameters $(\mathrm{mm})$

spindle rotation angle $\left({ }^{\circ}\right)$

nominal tool offset $(\mathrm{mm})$

depth of cut of each grain $i(\mathrm{~mm})$

maximum depth of cut (mm) 


\section{1) Introduction}

Polishing is a common finishing stage of advanced workpieces. Polishing operations, unlike grinding, use flexible tools and are employed to improve surface roughness without significantly modifying surface form. Thus, the control and prediction of the obtained surface roughness is an important issue for polishing operations. To predict and control the surface quality of polishing operations, a method has been developed in this work, to define a digital twin of polishing operation. In literature, numerous grinding and polishing digital twins have been implemented. Polishing and grinding are complex operations that involve multi-scale phenomena. The result of these processes is the outcome of a multitude of local cutting phenomena performed by grains upon material. In order to devise digital twins of finishing operations (polishing or grinding), three types of methods can be distinguished in literature:

- Empirical methods: they are based on a mathematical model best-fitted by experimental results. For example, Moumen et al. (2016) study flank polishing operations with abrasive caps using a 5-axes milling machine. By means of experimental testing, they obtain an empirical model able to reduce polishing cost by optimising polishing parameters (spindle speed, feed rate, toolpath shape, radial engagement, and grain size). Zhao et al. (2014) perform single-factor belt polishing experiments to build an empirical model in order to predict surface roughness and obtain optimum process parameters in belt polishing (abrasive size,

100 contact force, belt linear velocity and feed rate). The use of empirical models is restricted to the interpolation of the tested parameters for the process in question.

- Semi-empirical methods: they use an elementary experimental result that is analytically moved along a toolpath to predict the overall result. These methods use experiments to determine the behavioural law of a physical phenomenon and then, implement this law in a digital twin. For instance, Cheung et al. (2011) experimentally characterise the Tool Influence Function (TIF), which is the footprint left by a tool during a punctual contact with a surface. Thereafter, following their method, this TIF is displaced along a toolpath to virtually cut the surface and thus predict the obtained surface topology. Lu et al. (2019) used a TIF

110 experiment in dual-axis wheel polishing to derive the roughness topology in cases where either a single or dual-axis process was used. Thereafter, the obtained result was translated along the toolpath to predict the final surface topology. Through this methodology, they concluded that dual-axis polishing homogenises and reduces the generated surface roughness compared to single-axis wheel polishing.

- Analytical methods: they try to model the physical phenomena. These methods model cutting phenomena. Although they require more computing time, these kinds of models have more extensive applicability than empirical or semiempirical models. Results can be transferred to more than one polishing condition.

120 Among all grinding and polishing models described in literature, three levels of analytical models are differentiated (see Fig. 1):

- Nominal tool shape models: the nominal geometry of the tool leaves its shape's footprint on the surface. These models are useful only for grinding methods since tool grains are not considered. Denkena et al. (2010) develop this kind of model in order to predict surface roughness in 5-axes grinding operations. Zhang et al. (2020) used this type of model to predict the profile roughness of internal tubes finished by a novel magnetic polishing process. 
- Tool envelope profile models: a 2D profile is defined to simulate the tool envelope considering the tool grains. This profile is frequently used to predict surface roughness. This type of model is often more accurate than nominal tool shape methods in predicting surface roughness but is not suitable for waviness and form aspects. Li et al. (2020) develop this kind of model to predict the surface morphologies and surface roughness in the grinding of gallium gadolinium garnet single crystals. In order to achieve a more realistic and accurate determination of the tool envelope profile, $W u$ et al. (2020) propose to measure this profile while the tool is mounted on the machine. This measure is carried out using a laser displacement sensor system. Uhlmann et al. (2016) consider this type of model to predict surface roughness in 4-axes grinding processes.

- Models that consider each grain: cutting phenomena of every tool grain are considered. All these local phenomena are added to get macroscopic results of surface roughness. Darafon et al. (2013) carry out a method that takes each grain into account and determines uncut chip thickness, instantaneous material removal rate, contact length and surface roughness. They consider a spherical geometry of grains and a stochastic abrasive wheel model. Agarwal and Khare (2004) develop the same kind of digital twin considering spherical grains and a random distribution of grain protrusion heights. They obtain a relationship between surface roughness and chip thickness. In the grinding method proposed by Zhou et al. (2002), wear is taken into account by truncation of a Gaussian distribution model. The wheel wear volume is related to the change in the mean value of protrusion heights

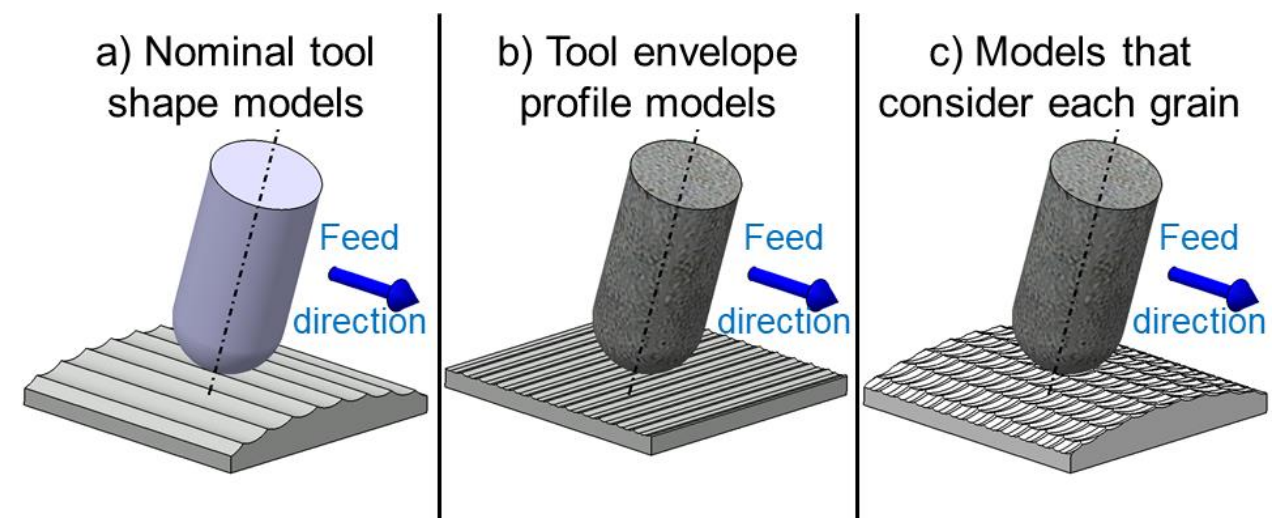

Fig. 1. Three types of analytical model to predict the roughness

Polishing digital twins are uncommon in literature and numerous papers propose methods without taking into account tool flexibility. Accounting for vibration is a first step in this direction. Jamshidi et al. (2019) take into account the vibrations in a model that considers each grain to identify the active ones on a wheel. Thereafter, this model was completed to predict the grinding forces (Jamshidi et al. (2020)). Xi and Zhou (2005) develop a method considering each grain to predict surface roughness, based on the micro depth of cut calculated by Brinell theory. They determine the number of contact grains and the Gaussian probability function of grains in the tool from a given stone grit number. Wang et al. (2009) consider Hertzian pressure at the contact site between tool and workpiece in their method which calculates material removal values 
of all abrasive grains that cut. The elastic deformation of the tools is considered in the polishing method developed by Qi et al. (2016), but this study is limited to the prediction of the material removal rate and does not include surface roughness determination. It follows Hertz elasticity contact theory and Brinell plasticity theory, and it assumes conic abrasive grain shape with spherical tip.

To conclude this overview from literature, there are very few models that consider each grain and tool flexibility, and none of them predicts the surface roughness. Thus,

175 this paper presents an Analytical-Method for Polishing-Surface Prediction (AMPSP) that considers the tool flexibility, the initial surface and each grain-material interaction. The objective is to predict accurately the surface topography (to be able to identify all roughness criteria), the material removal rate, and to keep a history of all local interactions. In section 2, the AMPSP is presented and each computation step is

180 detailed. In section 3, experimental tests are presented, to validate the mechanical model of tool/surface contact, the predicted material removal rate as well as the predicted surface topology.

\section{2) AMPSP: Analytical-Method for Polishing-Surface Prediction}

185 The main objective of the AMPSP is to predict analytically the topography of the resulting surface for polishing operations. Inputs of the AMPSP are presented in Fig. 2:

- Tool:

- Nominal tool shape geometry.

- Grain size distribution.

$190 \quad \circ$ Macroscopic mechanical properties of the tool: Young's modulus $E_{T}$ and - Workpiece: Poisson ratio $v_{T}$. (see section 3.1)

- Mechanical properties of the workpiece: Young's modulus $E_{W}$ and Poisson ratio $v_{W}$.

$195 \quad$ Initial surface topography of the workpiece.

- Process parameters:

○ Toolpath.

- Spindle and feed velocities, respectively: $\mathrm{N}$ and $V_{f}$.

$\circ$ Nominal tool offset. Usually this data is a common cutting parameter in polishing CAM software. This value is only used to help the operator estimate the suitable discretization step of the surface (see section 2.2). However, the real tool offset is calculated during the AMPSP process at each time step (see section 2.4.3).

Using provided inputs, the AMPSP carries out a simulation and gives as output the 205 predicted surface topography of the polished surface. In Fig. 2, inputs and outputs of the AMPSP are presented schematically. Fig. 3 summarises the successive processing steps in the polishing method. The upper part of this chart shows the different inputs and their distribution through the different computational steps. The processes outlined in this flowchart are detailed in the subsequent sections. 


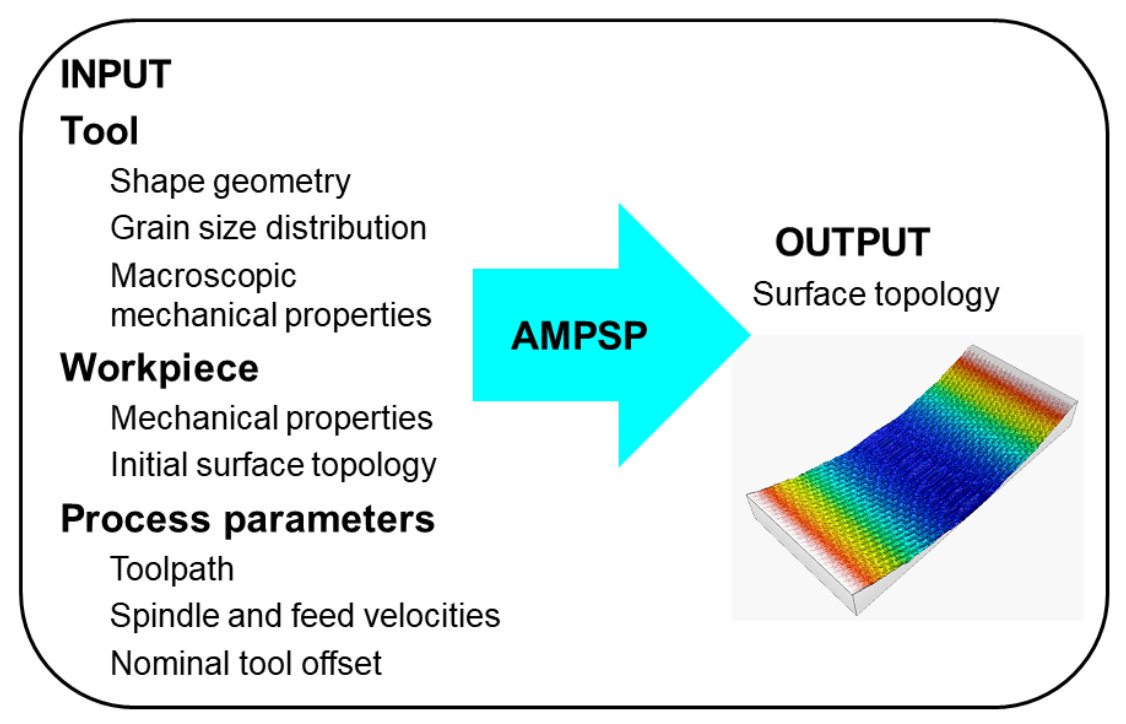

Fig. 2. Inputs and outputs of the AMPSP.

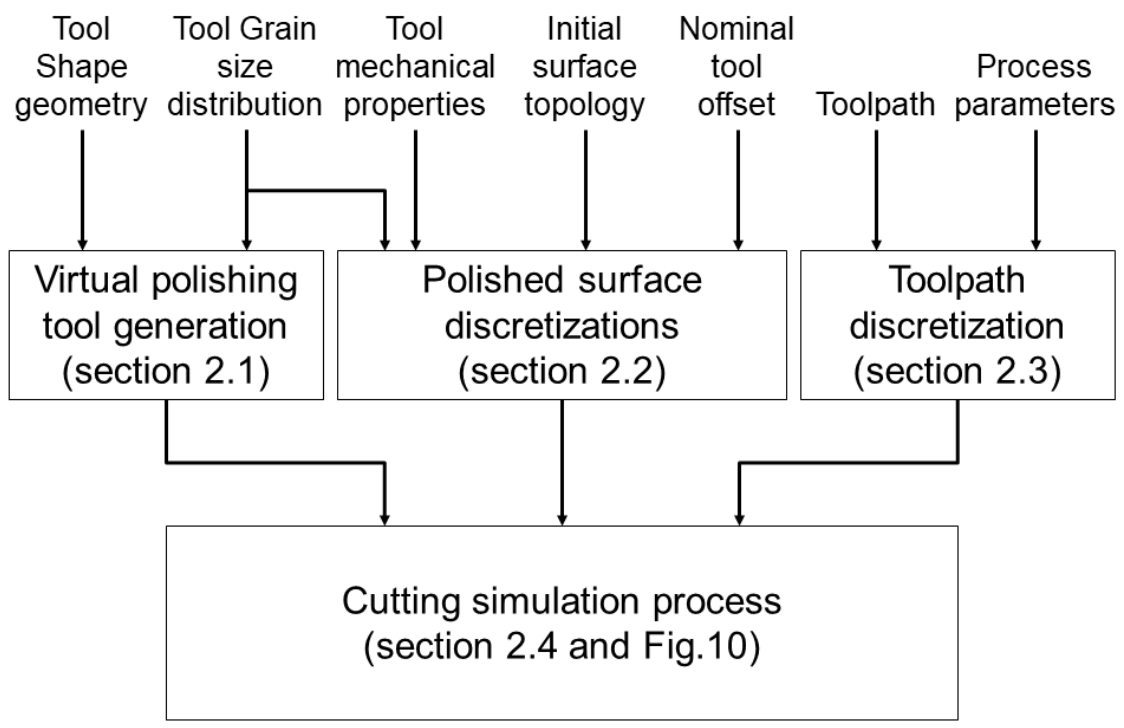

Fig. 3. Flowchart of the proposed method

\section{1) Virtual polishing tool generation}

In AMPSP, the tool is defined by a cloud of grains which may be distorted by the tool-surface contact. In this section, the steps used to generate the virtual tool are 220 detailed. Firstly, the nominal tool shape is defined. Secondly, models are proposed to determine the grain distribution in relation to the standard grit number. Finally, the virtual tool is built by randomly positioning grains on the tool nominal surface.

\subsection{1) Nominal tool shape generation}

225 The first step for the tool shape generation is to define a tool profile that will be rotated around its axis. For that, a generic tool profile is proposed. Its geometry depends on two parameters, the major torus radius $R$ and the minor torus radius $r$ (Fig. 4 a). These parameters allow the generation of main tool geometries (Fig. 4 b). 
(a)

(b)

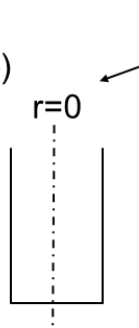

Flat end tool
Ball end tool

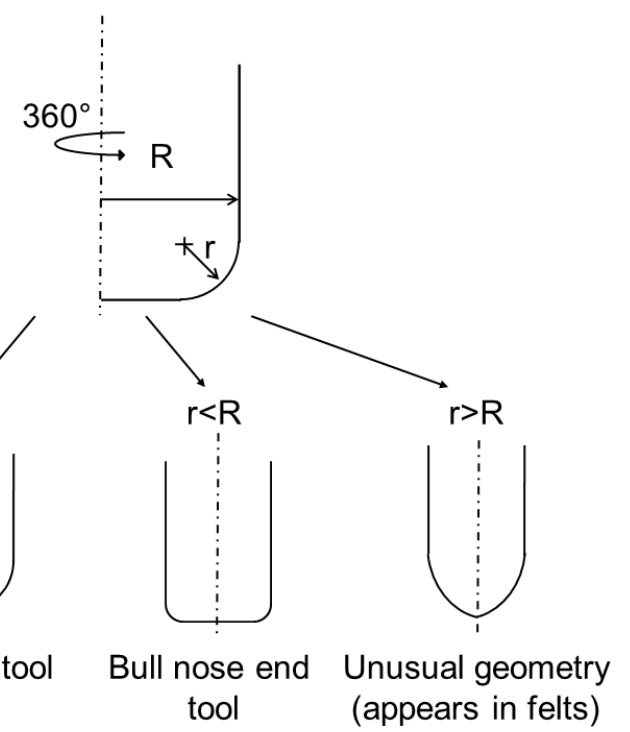

Fig. 4. (a) Proposed generic tool profile, (b) main tool geometries.

\subsection{2) Grain size model based on standard grit number (Tissier (2001))}

235 Once a tool profile geometry has been established, the tool's grain population and shape have to be defined. Malkin et al. (2008) demonstrate that the grain shape has a great influence on the surface roughness of the workpiece. In AMPSP method, the surface is discretised using a grid, which size is close to the footprint of a grain (see section 2.2). This discretization limits the effects of the chosen grain shape on the results. Thus, in the present work, the abrasive grains of the tool are modelled as spheres. With this model, the tool grains are defined by the position of their centre and their diameter.

In addition, to specify the grain diameter population on a tool, a model was proposed. It is based on standard grit numbers ISO 6344-1, 2 and 3 (Tissier (2001)). For each grit number, standards provide percentages of grain population that remain in several sieve sizes. Many grinding and polishing digital twins developed in literature use Gaussian distribution to model tool grain population (Xi and Zhou (2005), Wang et al. (2009)). Here, this kind of distribution is also employed. The grain distribution, associated to the grit number, is defined by (Fig. 5):

- $D_{\text {mean }}$. It represents the mean diameter of the grains for each given grit number, as defined by standard.

- $\sigma$. It represents the standard deviation of Gaussian model in grains' diameter.

- $D_{\max }$. It is the maximum grain diameter defined by standard. It is used to truncate the Gaussian grain distribution which is nominally infinite. 


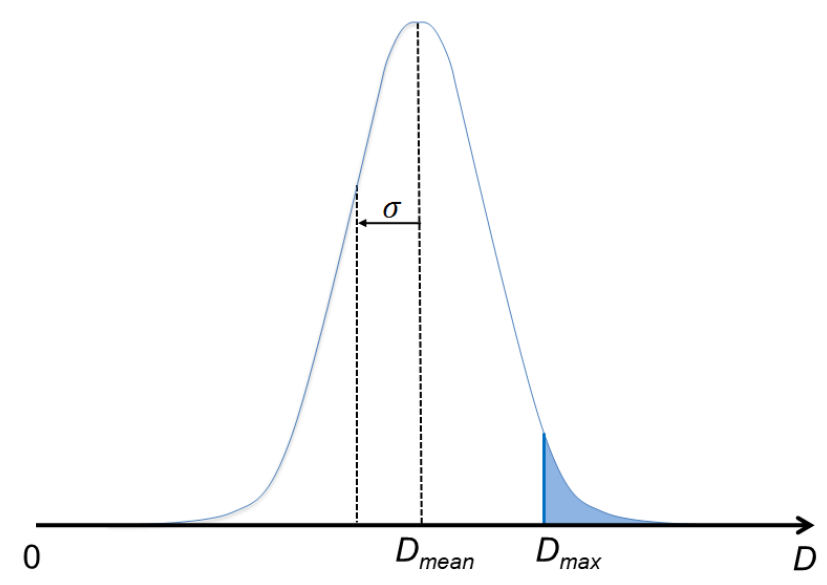

Fig. 5. Gaussian model of grain size distribution.

Eqs. 1-6 are proposed as best-fit values of $D_{\max }, D_{\text {mean }}$ and $\sigma$, provided by the 260 standards (Tissier (2001)). The proposed model takes into account grit numbers for macro-grains from \#60 to \#220 and micro-grains from \#240 to \#1200. The equations of the model were obtained by best fitting the values given by standard. For each standard grit number $N_{\text {grain }}$, values of $D_{\max }, D_{\text {mean }}$ and $\sigma$ can be obtained for both macro-grains (Eqs. 1-3) and micro-grains (Eqs. 4-6). To illustrate the relevance of proposed best-fit, 265 the curve defined by eq. 5 (grey line) and the values defined by the standard ISO63441,2,3 (black spots) are plotted in Fig. 6. This figure highlights a suitability of the proposed model.

$$
\begin{gathered}
D_{\text {max }}=\frac{15}{N_{\text {grain }}}+4.67 \times 10^{-2}(\mathrm{~mm}) \\
D_{\text {mean }}=\frac{13}{N_{\text {grain }}}+4.6 \times 10^{-3}(\mathrm{~mm}) \\
\sigma=\frac{1.5}{N_{\text {grain }}}+4.4 \times 10^{-3}(\mathrm{~mm}) \\
D_{\text {max }}=\frac{31}{N_{\text {grain }}}-2.6 \times 10^{-2}(\mathrm{~mm}) \\
D_{\text {mean }}=\frac{17}{N_{\text {grain }}}-1.5 \times 10^{-2}(\mathrm{~mm}) \\
\sigma=\frac{1.5}{N_{\text {grain }}}-1.2 \times 10^{-3}(\mathrm{~mm})
\end{gathered}
$$

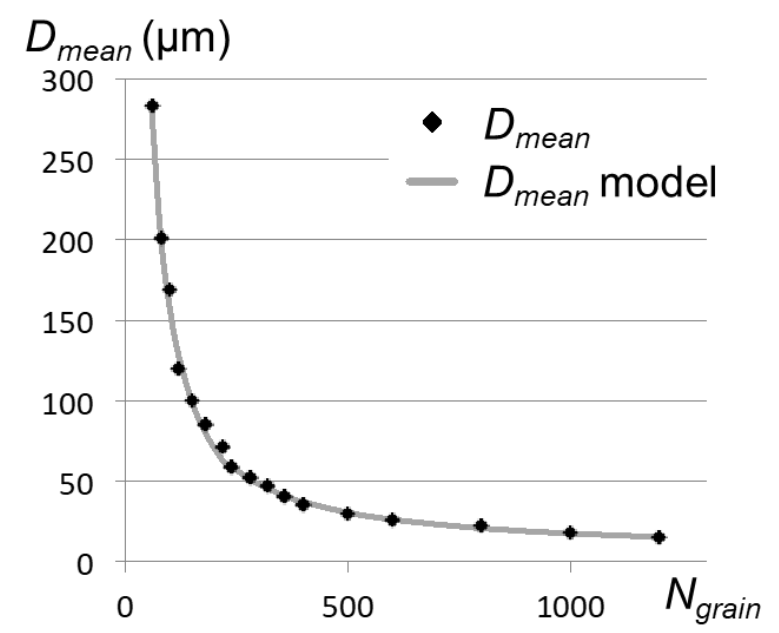


Fig. 6. $D_{\text {mean }}$ defined by standard grit number (Tissier (2001)) and proposed interpolation.

\subsection{3) Tool generation}

As seen previously, grains are modelled as spheres. A revolution of the tool nominal profile is performed to obtain the tool nominal surface. Thereafter, as proposed by $X i$ and Zhou (2005), the centres of the grains are aligned along the nominal tool. Thus, the centres of the spheres which model the grains are positioned on the tool nominal surface and a grain diameter $\left(D_{i}\right)$ is randomly selected using the distribution as defined in section 2.1.2. Each centre is randomly positioned, and a 3D collision test is carried out with all previously positioned grains. If no collision is detected, the position of the grain is validated, otherwise, the grain is removed. In this model, only the active grains are represented. The density of these grains depends on the tool type. Chaves-Jacob et al. (2021) propose an experimental procedure to determine this density. Fig. 7 represents an example of a tool generated in the AMPSP. In the AMPSP, only the cutting zone of the tool is generated in order to reduce computing time.

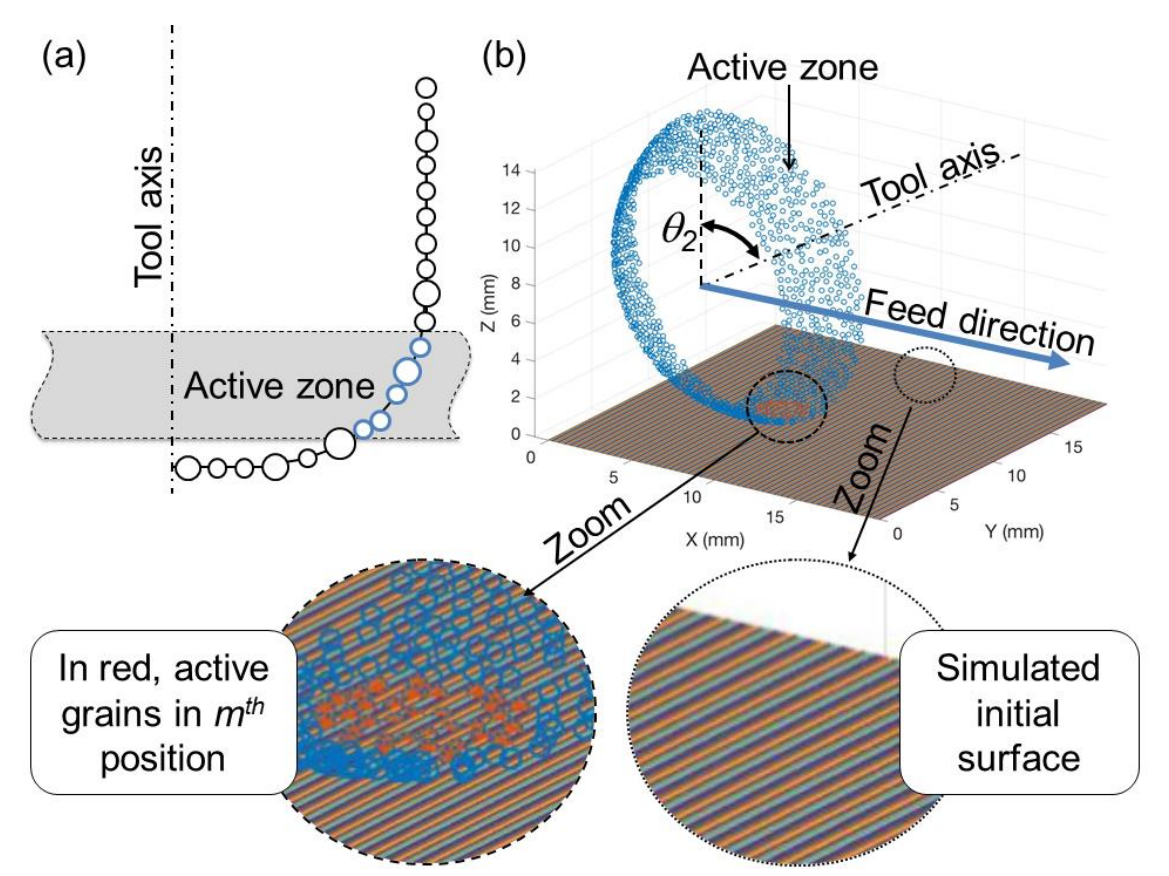

Fig. 7. (a) Grain placement in tool profile, (b) generated tool example in 3D.

\section{2) Polished surface discretization}

In the AMPSP, the surface of the workpiece is discretised using a grid (see Fig. 8 which defines the flowchart used to determine the grid size). The definition of the grid size is a trade-off between the predicted surface accuracy and the computing time. Here, the grid size, $\Delta_{\text {grid }}$, is selected to be equal to the average footprint computed using Brinell theory with a mean grain diameter, $D_{\text {mean }}$, and an average force applied on this grain, $F_{a}$. (Fig. 8 a). The average force is estimated using the applied nominal force $F_{N}$, determined with the nominal tool offset and Hertz theory. In order to compute the average force, the number of grains is estimated by dividing the nominal contact surface area (computed with Hertz theory) by the surface occupied by an average grain. Brinell theory is applied in Eq. 7 to estimate the mean grain's micro depth of cut and the grid size respectively. Thus, as presented in Fig. 8 b, in AMPSP, the surface is modelled as a succession of horizontal tiles which all have an independent altitude $Z_{x y}$. 


$$
\Delta_{\text {grid }}=4 \sqrt{\frac{D_{\text {mean }}}{2}-\left(\frac{D_{\text {mean }}}{2}-\frac{F_{a}}{D_{\text {mean }} \pi \mathrm{HB}}\right)^{2}}
$$

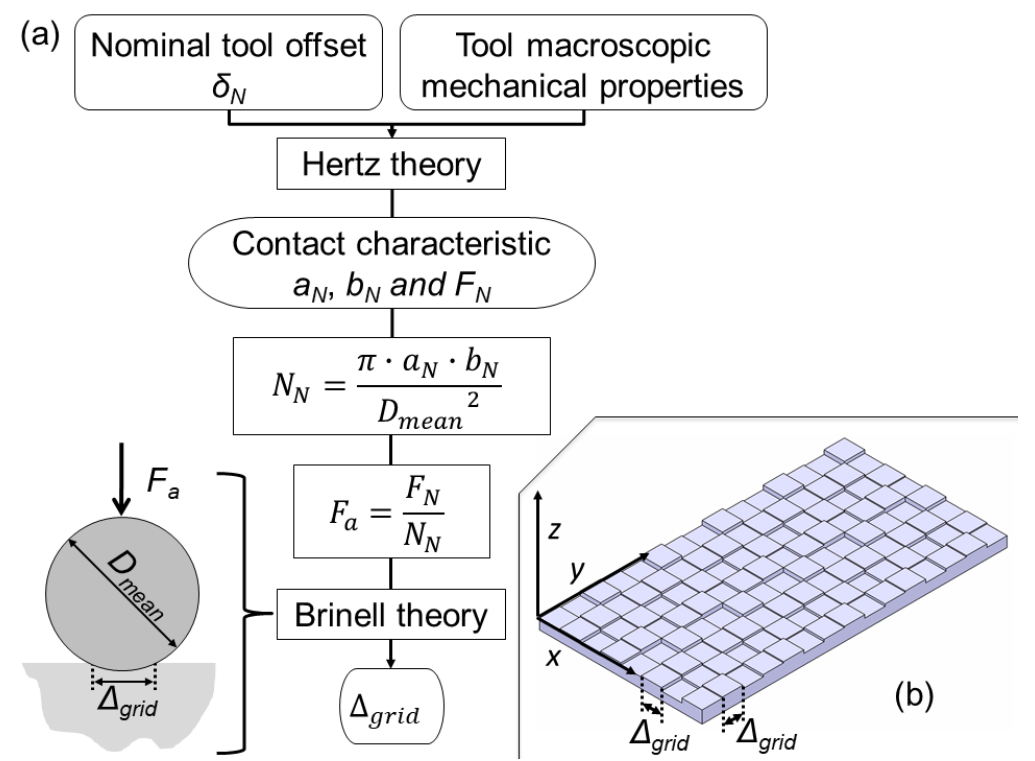

Fig. 8. (a) flowchart to determine the surface discretization size; (b) surface discretization grid.

\section{3) Toolpath discretization}

320 The AMPSP is developed for a 5-axes toolpath. Discretization of such a toolpath is required for the computation of the model. This discretization is once more a trade-off between predicted surface accuracy and computing time. In the proposed simulation, no interpolation is carried out between two simulated positions. To this end, the tool displacement between two simulated positions must be accurately controlled. To obtain 325 enough discretization, the peripheral displacement was fixed at a quarter of the value of the mean grain diameter, as illustrated in Fig. 9. This displacement corresponds to a spindle rotation of $\Delta \omega$. This angle was calculated considering the maximum tool radius $R$ and the mean diameter of grains (Eq.8). This spindle rotation angle discretization $\Delta \omega$ induces a time discretization $\Delta t$ that is calculated as a function of the spindle rotation 330 speed $N$ (Eq. 9).

$$
\begin{array}{r}
\Delta \omega=\tan ^{-1}\left(\frac{D_{\text {mean }}}{4 \cdot R}\right) \\
\Delta t=\frac{\Delta \omega}{60 N}
\end{array}
$$




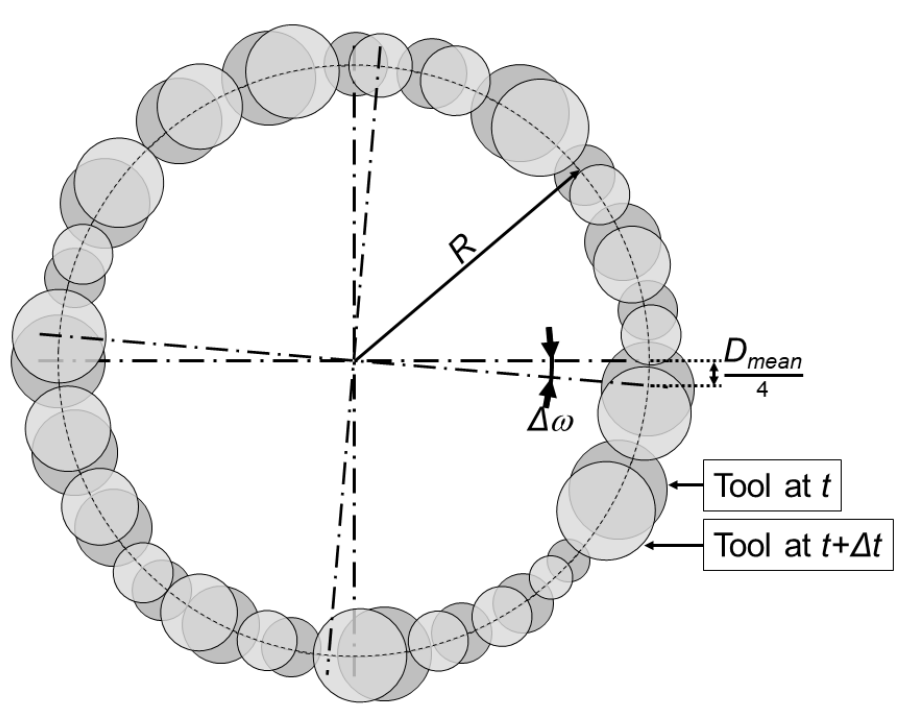

Fig. 9. Tool rotation discretization model.

\section{4) Cutting simulation process}

340 Fig. 10 illustrates the cutting simulation process and its steps at each discretised point of the toolpath. Cutting simulation ends when the sequence of discretised points of the toolpath reaches the last point. The following sections present the steps in detail.

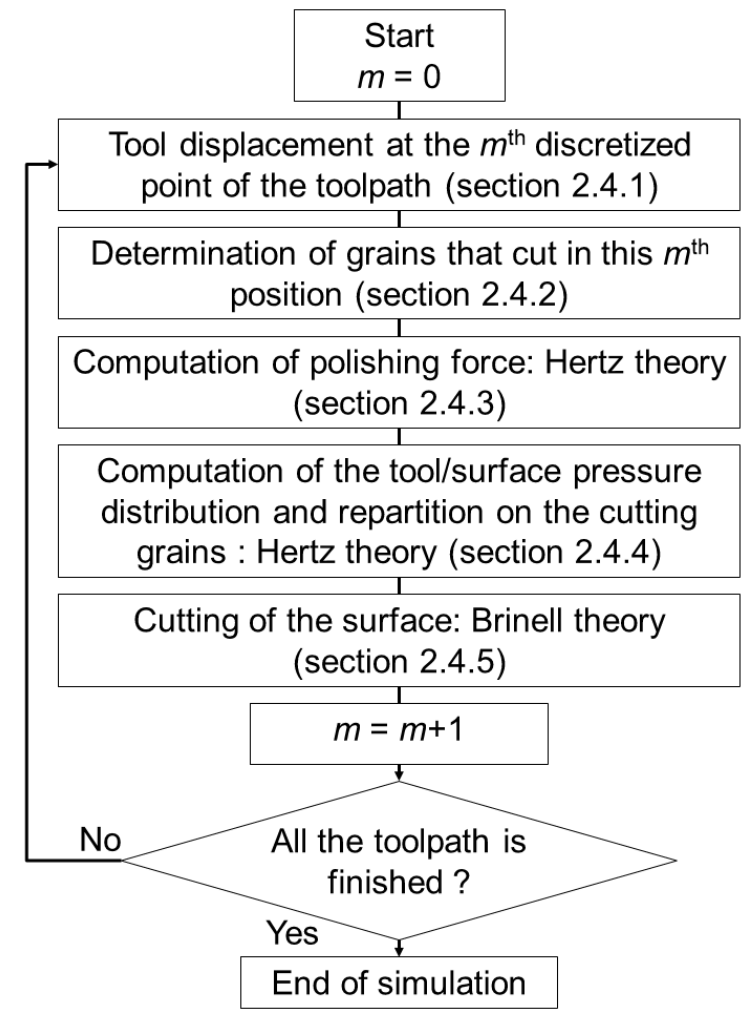

Fig. 10. Cutting simulation steps. 


\subsection{1) Tool displacement along the toolpath}

Section 2.3 presented the determination of time discretization of the simulated polishing operation. The AMPSP is able to predict the surface topology obtained by a 5axes toolpath. At each $m^{\text {th }}$ discretised point of the simulation, the tool position and orientation are determined using a linear interpolation between the previous and next points of the simulated toolpath. In the simulation, the tool is modelled as a cloud of grains (see section 2.1.3). When the position of the tool and its axes orientation for the $m^{\text {th }}$ point are determined, the cloud of points is translated and rotated according to this location. This operation allows the simulated tool to be set in the $m^{\text {th }}$ position.

\subsection{2) Determination of active grains in $m^{\text {th }}$ position}

In the AMPSP, the tool is considered a cloud of grains (see section 2.1.3). Once it is rotated and moved to the $m^{\text {th }}$ position, the grains that contribute to the cutting process in this simulated position must be identified. For that purpose, the depth $\delta_{i}$ of the grain $i$ in the simulated surface is calculated (Fig.11). Eq. 10 presents this computation. It is calculated by subtracting the height of the surface under the grain $i Z_{x y}$ from the lowest point of this grain noted $P_{b i}$. In this way, when $\delta_{i}$ has a negative value, the grain will cut the surface at this simulated point $\left(m^{\text {th }}\right)$ whereas when this value is positive, the grain will not touch the surface at this point.

$$
\delta_{i}=P_{b i}-Z_{x y}
$$

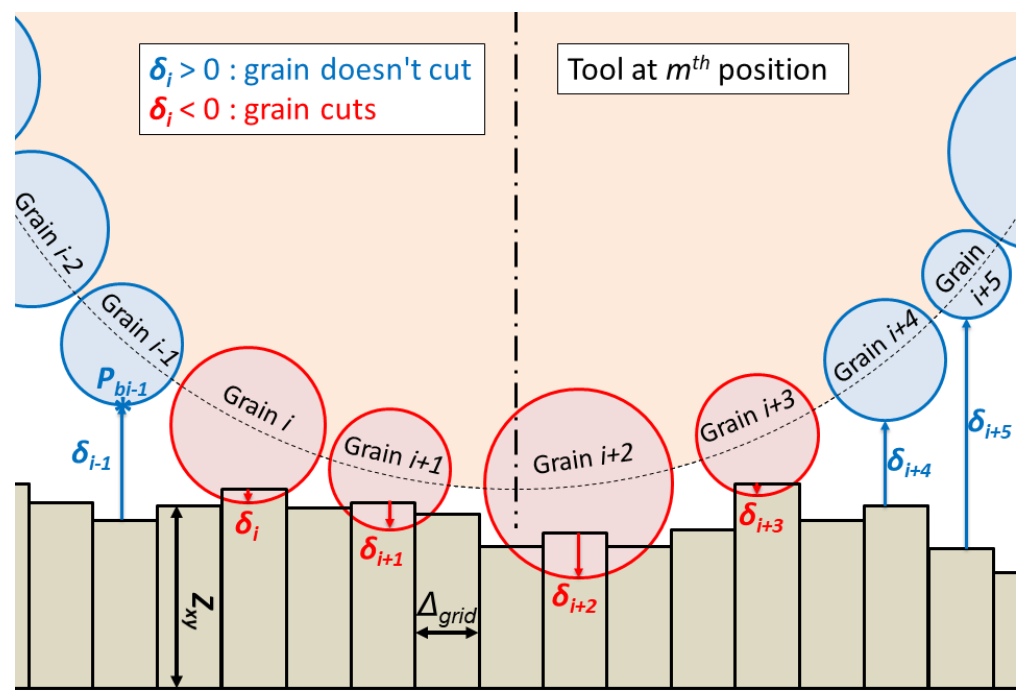

Fig. 11. Determination of grains that contribute to cutting process in simulated $m^{\text {th }}$ point.

\subsection{3) Computation of polishing force in $m^{\text {th }}$ position}

There is a wide variety of technologies available in tool polishing processes.

Four main tool families can be defined:

380 - Abrasive caps: sandpaper cap (covered by abrasives) is mounted on a flexible rubber support. These tools are used to remove wide volumes of material. They are generally used in rough polishing operations. However, these tools have a short lifetime. 
- Flexible wheels: in this case, abrasive grains are embedded into a supple rubber matrix. These tools have a better surface compliance than abrasive caps, but with a lower material removal rate. They are usually used in semi-finishing polishing stages.

- Felts: loose abrasive grains are applied on a soft polishing felt. These tools have a very high surface compliance, but material removal rate is very low. These tools are limited to the finishing polishing stage.

- Shape Adaptive Grinding (SAG) proposed by Beaucamp et al. (2015): this is a relatively new type of tool. Rigid pellets loaded with the abrasive grains are mounted on a flexible support. These tools have a high grinding ratio, and thus a long lifetime.

These four tool families involve different mechanical behaviours at the contact site between the tool and the polished surface due to the variety of physical phenomena. To take into account these differences in the AMPSP, different mechanical behaviours are considered. The Hertzian contact theory proposed by Hertz (1881) is used to model the elastic deformation of the tool (Fig.12). In this theory, the tool is considered a homogeneous material in the area of contact. Macroscopic Young modulus of tool $E_{T}$ and macroscopic Poisson's modulus of tool $v_{T}$ have to be determined. An experiment is proposed in section 3.1 to determine these values.

To estimate the force $F_{m}$ between the tool and the workpiece at the considered polishing time, the maximum value of depth $\delta_{\text {imax }}$ (computed in section 2.4.2) is used. The relation between force and depth is established with Hertz theory (Fig. 12).
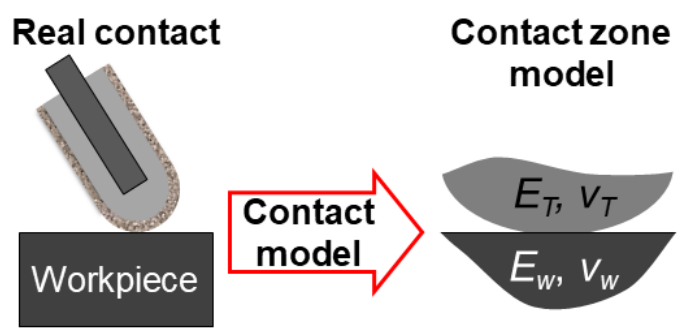
model
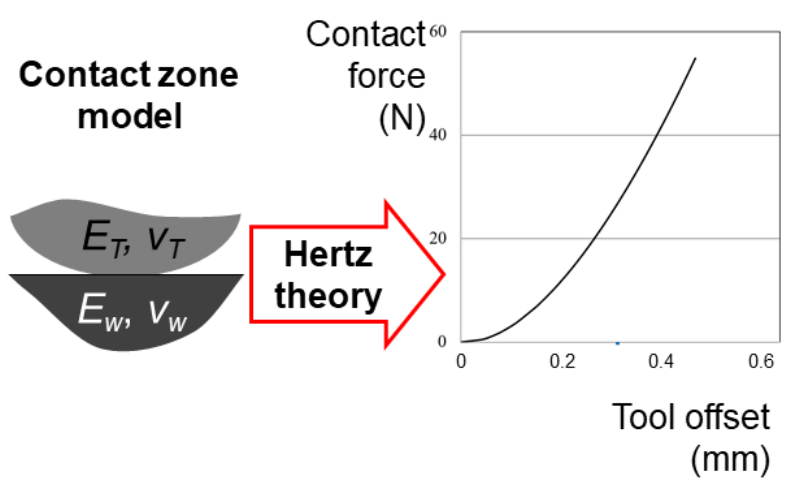

Fig. 12. Model of contact between the tool and the polished surface.

\subsection{4) Computation of the tool/surface pressure distribution and repartition on the cutting grains}

As proposed in Hertz theory, the contact ellipse between the tool and the workpiece is calculated using the contact force $\left(F_{m}\right)$ determined in section 2.4.3 and the tool's mechanical properties (see section 3.1). The contact ellipse is defined by major and minor semi-axes noted $a$ and $b$ respectively. Finally, Hertzian pressure $P_{0}$ (Eq. 11) and pressure field between the tool and the surface (Eq. 12) are calculated. In eq. $12 X_{i}$ and $Y_{i}$ represent the coordinates of the considered point in the local contact framework. This local contact framework is defined as follows: $\mathbf{X}$ and $\mathbf{Y}$ axes are respectively aligned with the minor and major semi-axis, the origin is centred on the contact area.

$$
P_{0}=\frac{1.5 F_{m}}{\pi a b}
$$




$$
P_{i}=P_{0}^{2} \sqrt{1-\left(\frac{X_{i}^{2}}{b^{2}}+\frac{Y_{i}^{2}}{a^{2}}\right)}
$$

Fig. 13 illustrates the method to split the applied forces (see section 2.4.3) onto each active grain. The pressure field is used to define the proportion of $F_{m}$ applied to each active grain. The pressure applied to each grain is derived from the position of this grain in the pressure field $P_{i}$ (Eq. 12). Then, the proportion of $F_{m}$ on each grain is computed into a proportional coefficient used to determine the effort applied on each grain (Eqs. 13-14).

430

$$
\begin{gathered}
F_{i}=\frac{F_{m} \times P_{i}}{\sum_{i} P_{i}} \\
\sum_{i} F_{i}=F_{m}
\end{gathered}
$$

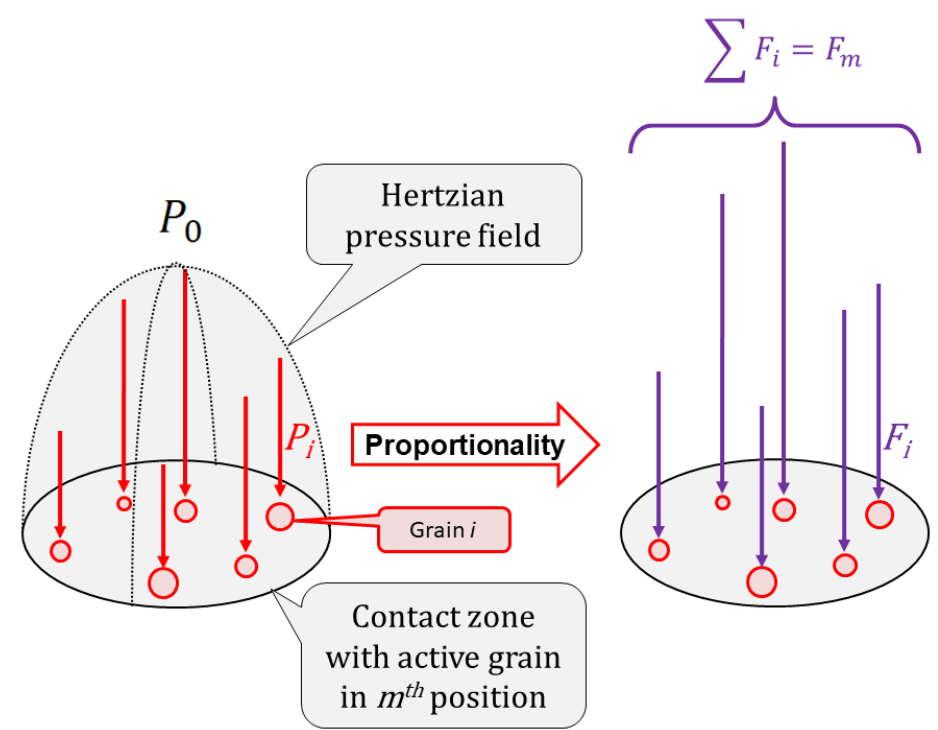

Fig. 13. Method to calculate the contact force on each active grain.

\subsection{5) Surface cutting simulation}

$440 \quad$ Nguyen and butler (2005) simulated the interaction between the abrasive grain and the workpiece in grinding, considering the three cutting modes: cut, plough or rub. In this simulation the attack angle of grains is used to determine the adequate mode for each grain in the simulation. In a first approach, in AMPSP only the cutting mode is considered. In future work, the side ridges along the groove induced by rubbing mode, transferring material to neighbour cells of the discretised surface, could be taken into account. Thus, in this proposed method, the Brinell theory is used to consider the plastic deformation induced by the cutting phenomenon. The depth of cut of each grain inside the contact ellipse is obtained with that theory (Eq. 15). This depth varies depending on the force applied to each grain $F_{i}$ (see section 2.4.4), its diameter $D_{i}$ and Brinell

450 hardness number HB. Fig. 14 illustrates the cutting simulation process. Thereafter, the depth of cut, $h_{i}$, of each active grain is subtracted from the tiles below the centre of each grain.

$$
h_{i}=\frac{F_{i}}{D_{i} \pi \mathrm{HB}}
$$




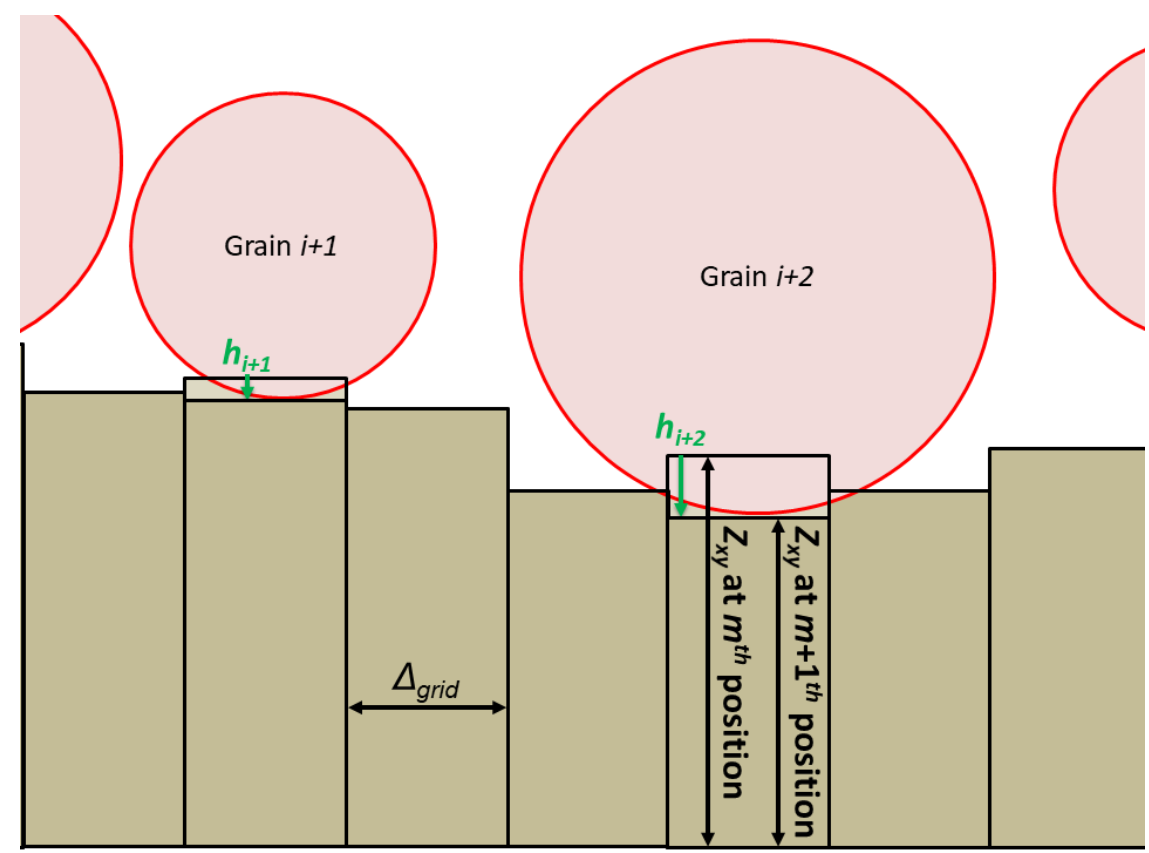

Fig. 14. Surface cutting model.

\section{3) Experimental validation}

\section{1) Experimental determination of method parameters}

As presented in section 2.4.3, the contact between the tool and the polished workpiece is modelled using the Hertz theory. This theory requires the mechanical properties of both solids in contact: Young modulus and Poisson's ratio. For the workpiece, natural mechanical properties are used; for the tool, macroscopic mechanical properties are determined using an experiment. Thereby, in order to determine these two mechanical properties, an elementary sphere/plan experiment was conducted. The experiment consists in a plunge test without spindle rotation of the flexible tool on a metallic plane mounted on a dynamometer sensor (see Fig. 15). The tool is moved down in steps in the normal direction of the metallic plane for several steps. Between each step a timer is set without tool displacement to carry out force measurement. Thereafter, the experimental force/depth relation is established, and a Hertz model is best fitted. Thus, both optimised macroscopic mechanical parameters of the tool are determined.

In Fig. 15, the experiment was carried out with two different configurations:

- Fig. 15 a: a flexible wheel tool composed of abrasive grains embedded in a rubber matrix was set in a 3-axes configuration. Experiments were conducted with a grit number of \#240 (average grain size of $46 \mu \mathrm{m}$ ).

- Fig. 15 b: an abrasive cap composed of sandpaper with a resin bond and mounted on a rubber support, was used in a 5-axes configuration with an inclination of $50^{\circ}$. Experiments were conducted with a grit number of \#60 (average grain size of $280 \mu \mathrm{m}$ ).

Fig. 15 presents, for both tested tools, the force/depth curve obtained experimentally (points), and the Hertz best-fitted model (continuous line). Both experiments confirm that the Hertz theory is able to accurately model the complex contact between the tool and the polished workpiece within the depth range used in polishing (tool offset $<0.4$ $\mathrm{mm})$. 

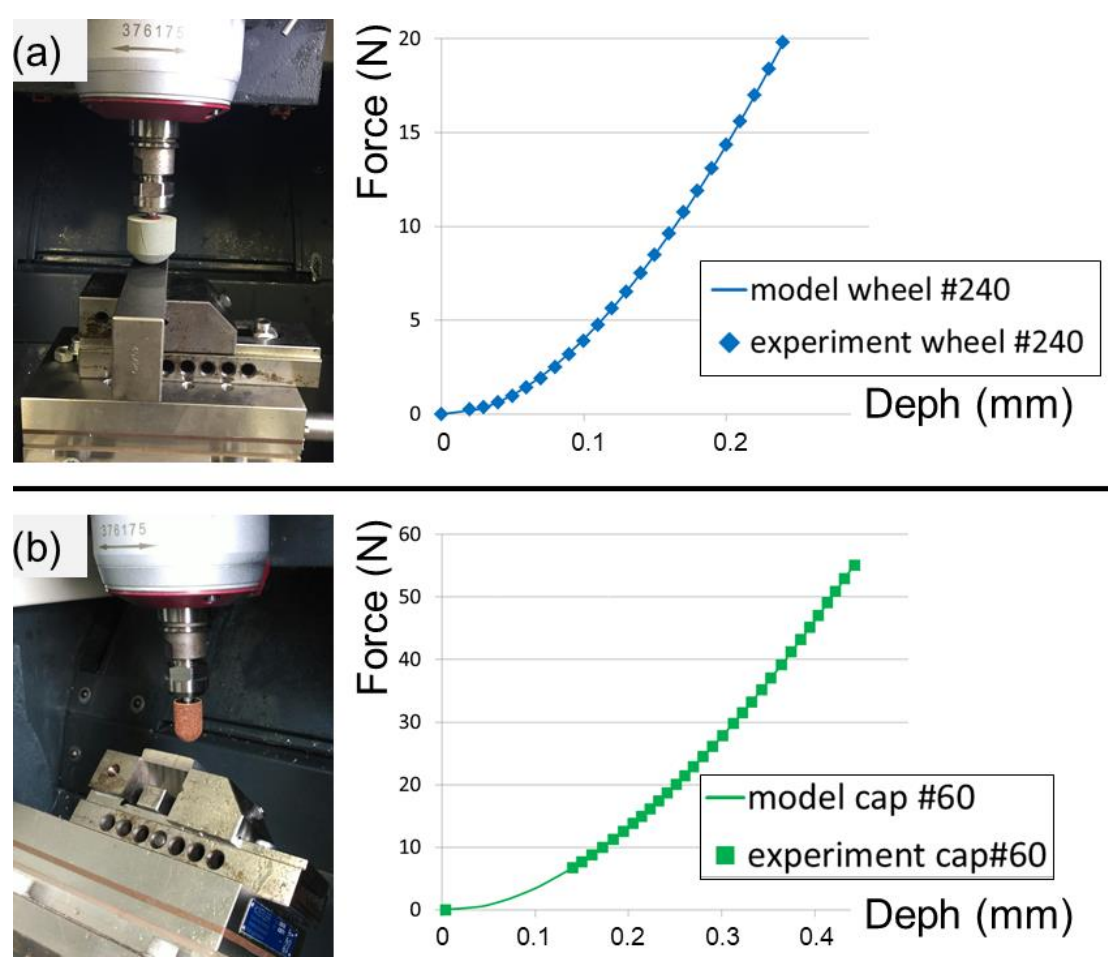

Fig. 15. Experimental determination of equivalent tool macroscopic mechanical parameters a) flexible wheel tool with a grit number of \#240 in a 3-axes configuration b) abrasive cap with a grit number of \#60 in a 5-axes configuration (inclination of $50^{\circ}$ ).

\section{2) Validation of material removal rate: single polishing line test}

To validate the material removal rate predicted by AMPSP a single polishing line test is carried out on a plane. This experiment consists in performing the polishing of a line on a plane (considered without defect). This experiment allows the depth of the realised groove to be measured accurately in relation to the reference plane surrounding it. 5 tests have been performed with different values of feed rate $V_{f}$, spindle rotation speed $N$ and tilt angle $\theta_{2}$. Polishing parameters and experimental elementary lines are shown in Fig. 16. Lines are $40 \mathrm{~mm}$ long and the workpiece is made of stainless steel 17-

5004 ph (elastic limit around 1,100 Mpa). For all the experiments, the employed type of tool was an abrasive cap with a spherical head of $8.5 \mathrm{~mm}$ of radius and a grit number of \#60. Experiments were carried out on a common 5-axes CNC milling machine. Used nominal tool offset was $0.3 \mathrm{~mm}$. For simulation, the virtual polishing tool was generated with grains set to fully fit the surface of the tool in order to represent the sandpaper (number of active grain). 


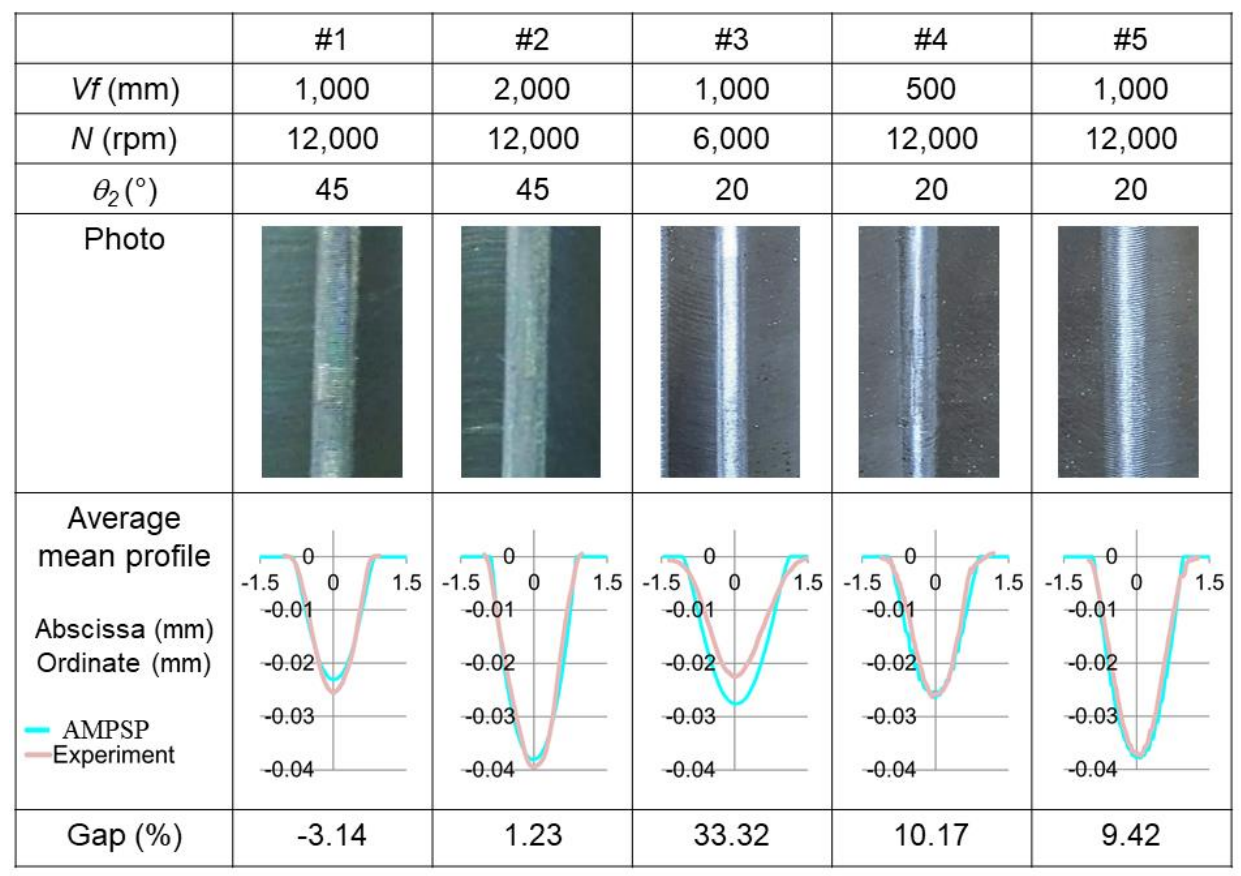

Fig. 16. Five experimental line tests to validate the predicted material removal rate of AMPSP.

Fig. 17 presents the measured surface topologies with experimental testing and the predicted surface topology by AMPSP for the line \#1. Experimental surfaces have been characterised through optical measurements using a chromatic confocal sensor CMM. The resolution of this CMM is about $10 \mathrm{~nm}$. The measurements were carried out on areas of $2.5 \mathrm{~mm} \times 1 \mathrm{~mm}$, centred on each line. The length of these areas was set perpendicularly to the direction of the lines and scanned in both directions with $4 \mu \mathrm{m}$ intervals. Presented results highlight the quality of the predicted surface topologies. Furthermore, to evaluate the accuracy of the predicted material removal rate, a mean of the profiles, both measured and simulated, was computed along $1 \mathrm{~mm}$ of the groove.

520 Both profiles are presented in Fig. 17 for the line \#1 and in the average mean profile row of Fig. 16 for the 5 performed tests. The gap (\%) row provides the percentage of gap between both lines. The result defines a qualitative projection of predicted material removal rate (gap < 35\%). 


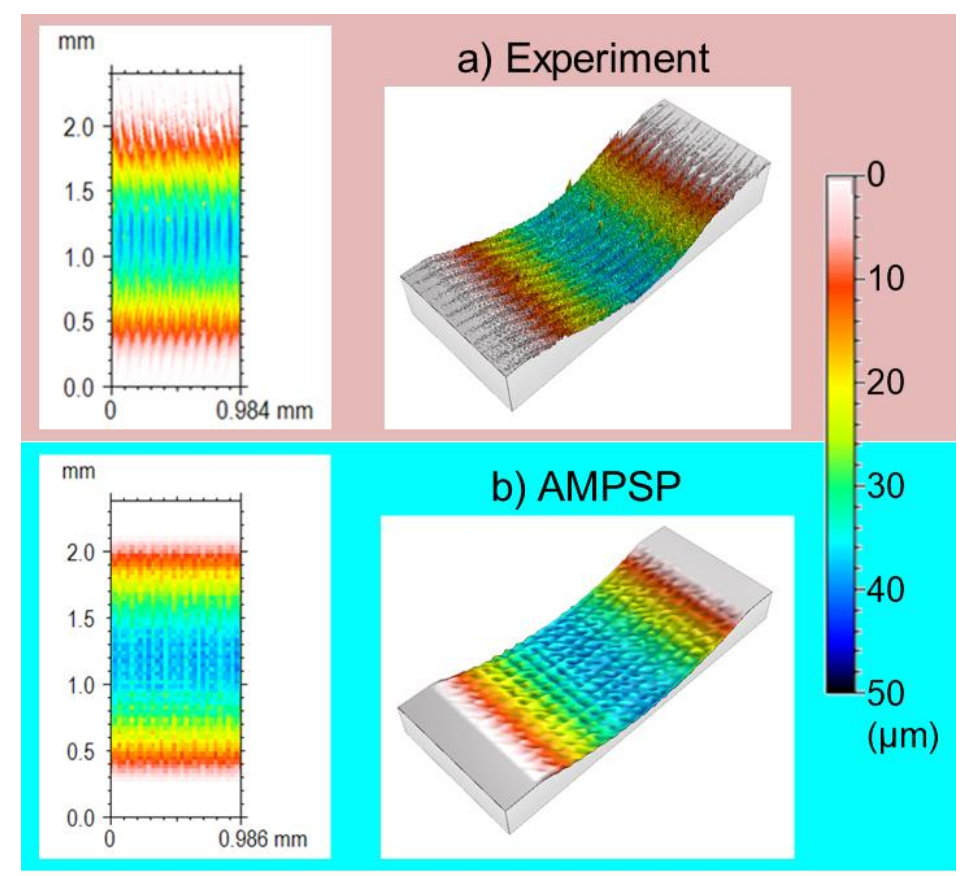

c) Average mean profile on $0.984 \mathrm{~mm}$

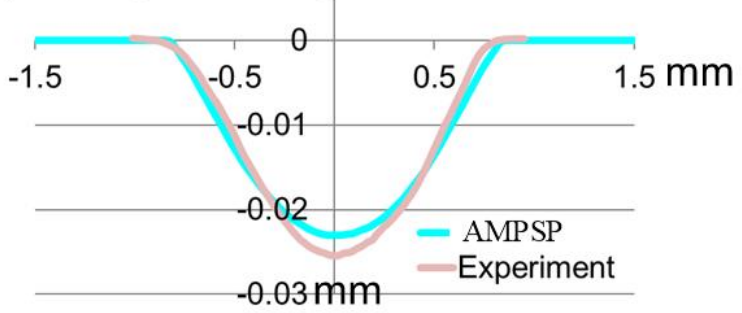

Fig. 17. a) Experimental \#1 measure b) predicted \#L1 measure c) mean profiles (predicted-blue line/ experimental-red line).

In order to validate the predicted topology of AMPSP, a surface polishing test was conducted. In this experiment, the surface of a workpiece made of stainless steel 17-4 ph and built by additive manufacturing was polished in the 5-axes CNC milling machine using a raster toolpath. Fig. 18 shows the workpiece before and after the 535 polishing experiment. The polished part of this workpiece is composed of a succession of three planes of $10 \mathrm{~mm}$ length, linked by corner radius. The tool used was an abrasive cap with a spherical head of $8.5 \mathrm{~mm}$ radius and a grit number of \#60 (average grain size of $280 \mu \mathrm{m}$ ). Polishing parameters were: a feed rate of $2000 \mathrm{~mm} / \mathrm{min}$, a spindle rotation speed of $12000 \mathrm{rpm}$, a tilt angle of $60^{\circ}$, a tool offset of $0.2 \mathrm{~mm}$ and a track spacing of $540 \quad 0.5 \mathrm{~mm}$.

The obtained surface was characterised through optical measurements using a chromatic confocal sensor CMM. As illustrated in Fig 19 and 20, the measured areas are three squares of $1 \mathrm{~mm}$ side located in the middle of the three polished planes. These three results are used to represent the experimental variability. Thereafter, a Gaussian

545 filter with a cut-off of $0.25 \mathrm{~mm}$ was used to separate the waviness and the roughness signal of the polished surface (Fig. 19 and 20). Then, arithmetical mean height (Sa) was computed on each surface. In parallel, a simulation was carried out using AMPSP to represent the experimental polishing operation. The predicted surfaces undergo the same treatment as the experimental surfaces. 


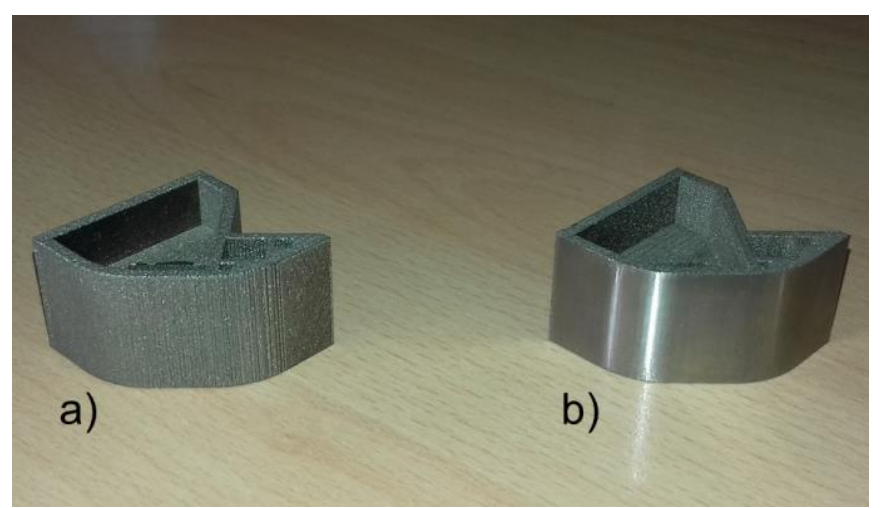

Fig. 18. Workpiece used to validate the obtained surface topology a) before polishing (rough of additive manufacturing) b) after polishing stage.

555 Waviness results (experimental and simulated) clearly highlight the successive parallel passes of the tool (from left to right in Fig. 19) separated by the values of lateral displacement between two passes $(0.3 \mathrm{~mm}$, in the height direction in Fig. 19). Experimental results present a small dispersion in surface topology, due to experimental variations (e.g., chatters or tool deflections). Apart from this mentioned variation, the predicted waviness is accurate in topology and values (gap between average values).

Roughness results (experimental and simulated) highlight scratches in the cutting speed direction (from top to bottom in Fig. 20). Furthermore, these results show a progressive reduction of the roughness as the polishing time passes (first machined surface, $\mathrm{Sa}=0.53 \mu \mathrm{m}$, second $\mathrm{Sa}=0.39 \mu \mathrm{m}$ and last $\mathrm{Sa}=0.23 \mu \mathrm{m})$. This evolution may be 565 explained by the fast tool wear of the abrasive caps. The tool wear directly modifies the grain distribution, as presented by Zhou and Xi (2002). The simulation performed by AMPSP (Fig. 20) was conducted with a digital "fresh tool" having a grain distribution as specified by the tool manufacturer. It seems logical that the performed simulation predicts a surface roughness close to the first polished surface $(\mathrm{Sa}=0.53 \mu \mathrm{m})$, where the tool was the newest.

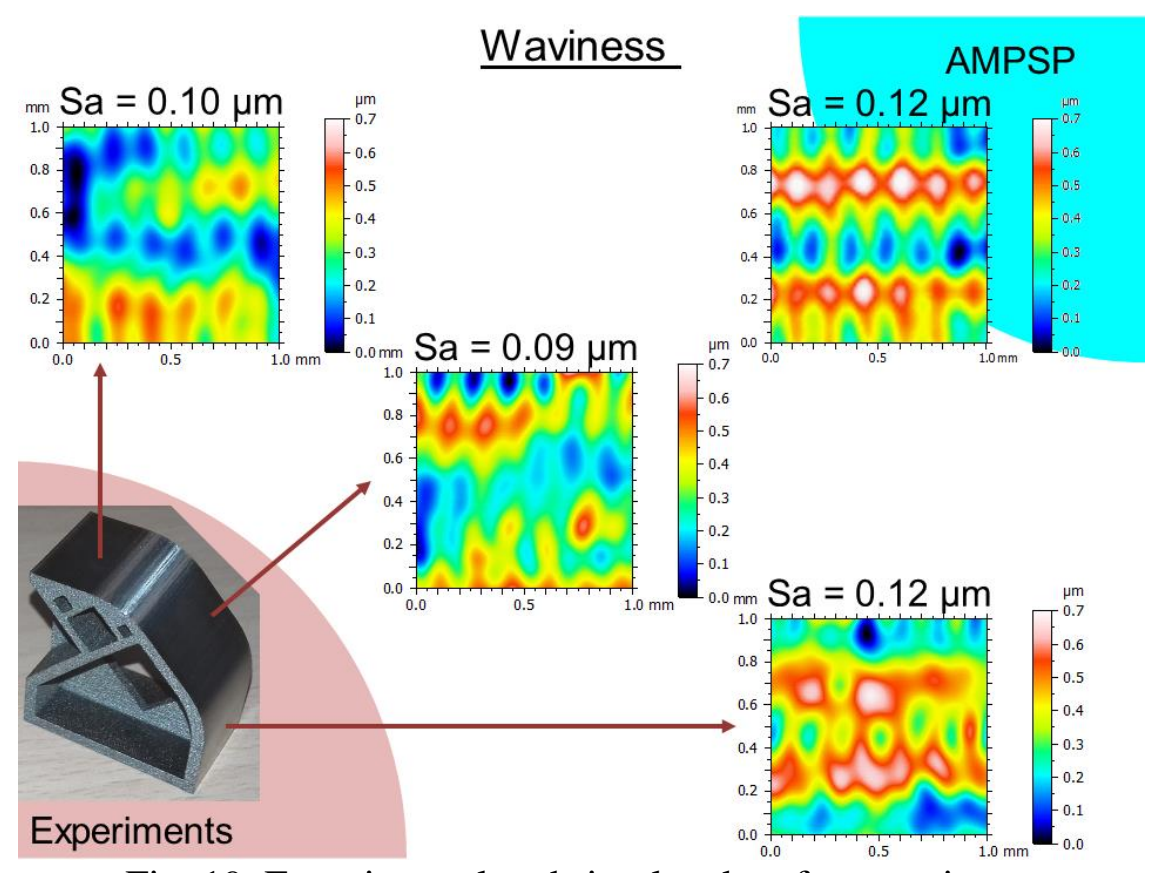

Fig. 19. Experimental and simulated surface waviness. 


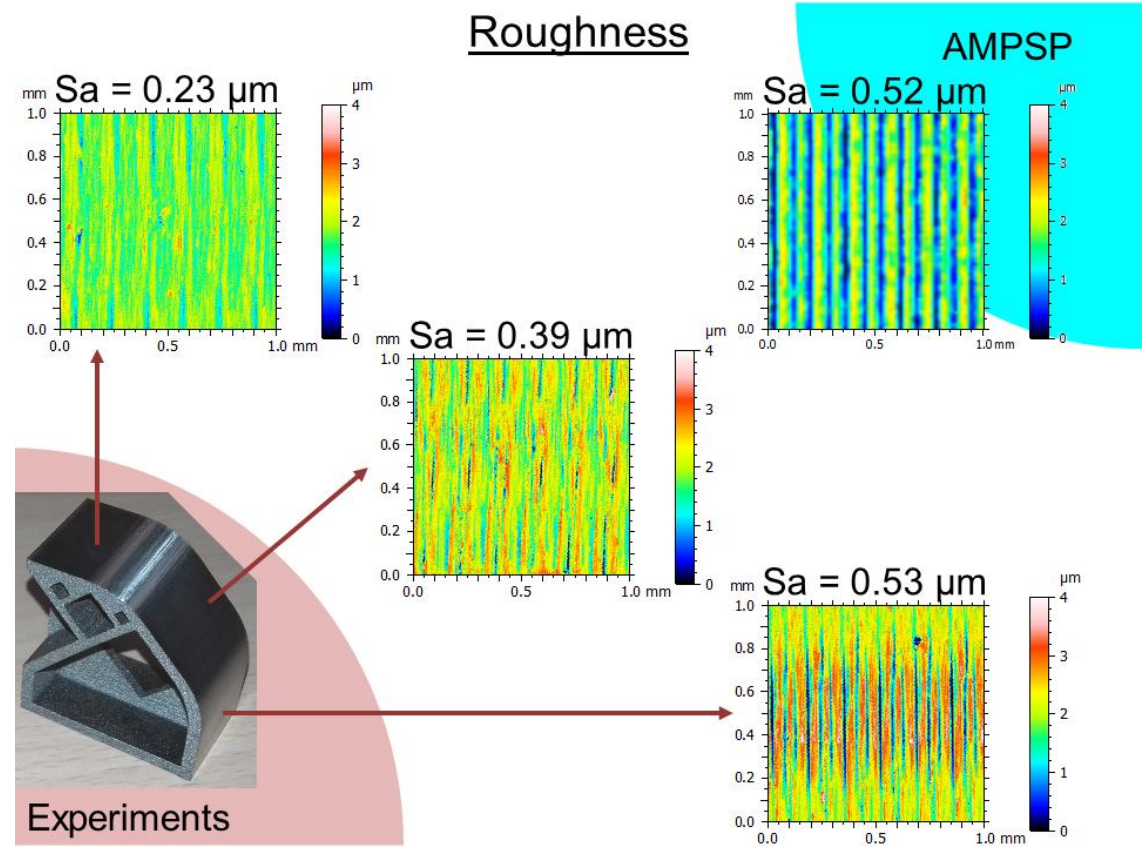

Fig. 20. Experimental and simulated surface roughness.

\section{4). Conclusions}

This work defines an Analytical-Method for Polishing-Surface Prediction (AMPSP) in order to control and predict results of polishing operations. The AMPSP is useful for 5 -axes toolpaths and is a digital twin of polishing operation. It considers the cutting process of each grain of the polishing tool. In AMPSP, the tool is defined as a cloud of grains which may be distorted by the tool-surface contact, and the surface of the workpiece is discretised using a grid (which size is suitably selected). Local cutting

585 phenomena are calculated with Brinell theory, and macroscopic contact between the tool and the surface is modelled with Hertz theory. The association of these two theories allows for a suitable multi-scale model of polishing phenomena. Experimental validation was conducted in three steps:

- Validation of the tool/surface contact model for two polishing tool-families.

$590-$ Validation of the material removal rate in five different polishing configurations (less than $35 \%$ of error).

- Validation of the predicted surface topology in waviness and roughness (less than a few percent).

One of the main limits of the AMPSP is the computation time (for example, the simulation of a $10 \times 10 \mathrm{~mm}$ surface using a tool with an average grain of $280 \mu \mathrm{m}$ needs around $48 \mathrm{~h}$ of computation on a common laptop). The time consumption cost of computation ought to be compared to the cost needed to obtain the same result experimentally. The ratio between both costs is in favour of the AMPSP. To conclude, AMPSP will enable engineers to predict the polished topology obtained with 5-axis toolpaths.

\section{5) Acknowledgements}

The experimental equipment was funded by the European Community, French Ministry of Research and Education and Aix-Marseille Conurbation Community. 
Agarwal and Rao (2004) Agarwal, S., Rao P.V. 2004. A probabilistic approach to predict surface roughness in ceramic grinding. International journals of machine tools and manufacturing. 1-8.

Beaucamp et al. (2015) Beaucamp, A., Namba, Y., Charlton, P., 2015. Process 610 mechanism in shape adaptive grinding (SAG). Annals of the CIRP. 64, 305-308.

Chaves-Jacob et al. (2021) Chaves-Jacob, J., Beaucamp, A., Zhu, W., Daisuke Kono, D., Linares, J.M. 2021. Towards an understanding of surface finishing with compliant tools using a fast and accurate simulation method. International Journal of Machine Tools \& Manufacture. 163, 103704

615 Cheng et al. (2011) Cheung, C.F., Kong, L.B., Ho, L.T., To, S., 2011. Modelling and simulation of structure surface generation using computer controlled ultra-precision polishing. Precision engineering. 35, 574-590.

Darafon et al. (2013) Darafon, A., Warkentin, A., Bauer R., 2013. 3D metal removal simulation to determine uncut chip thickness, contact length, and surface finish in 620 grinding. Orthopaedics and trauma. 66, 715-1724.

Denkena et al. (2010) Denkena, B., de Leon, L., Turger, A., Behrens, L., 2010. Prediction of contact conditions and theoretical roughness in manufacturing of complex implants by toric grinding tools. International Journal of Machine Tools \& Manufacture. 50, 630-636.

625 Hertz (1881) Hertz, H., 1881. Ueber die beruhrung fester elastischer korper. Journal reine und angewandte Mathematik. 92, 156-171.

Jamshidi et al. (2019) Jamshidi, H., Gurtan, M., Budak, E. (2019) Identification of active number of grits and its effects on mechanics and dynamics of abrasive processes. Journal of Materials Processing Tech. 273, 116239.

630 Jamshidi et al. (2020) Jamshidi, H., Budak, E. (2020) An analytical grinding force model based on individual grit interaction. Journal of Materials Processing Tech. 283, 116700.

Li et al. (2020) Li, C., Wu, Y., Li, X., Ma, L., Zhang, F., Huang, H., 2020. Deformation characteristics and surface generation modelling of crack-free grinding of 635 GGG single crystals. Journal of Materials Processing Tech. 279, 116577.

Lu et al. (2019) Lu, A., Jin, T., Liu, Q., Guo, Z., Qu, M., Luo, H., Han, M. (2019) Modeling and prediction of surface topography and surface roughness in dual-axis wheel polishing of optical glass. International Journal of Machine Tools and Manufacture. 137, 13-29.

640 Malkin et al. (2008) Malkin, S., Guo, C., 2008. Grinding Technology: Theory and Application of Machining with Abrasives, second edition. Industrial press, New York.

Moumen et al. (2016) Moumen, M., Chaves-Jacob, J., Bouaziz, M., Linares, J.M., 2016. Optimization of pre-polishing parameters on a 5-axis milling machine. Int. J. Manuf. Technol. 85, 443-454.

645 Nguyen and Butler (2005) Nguyen, T.A., Butler, D.L., 2005. Simulation of surface grinding process, part 2: interaction of the abrasive grain with the workpiece. International Journal of Machine Tools \& Manufacture. 45, 329-1336.

Qi et al. (2016) Qi, J., Zhang, D., Li, S., Chen, B., 2016. A micro-model of the material removal depth for the polishing process. Int J Adv Manuf Technol. 86, 27596502770.

Tissier (2001) Tissier, 2001. Norme française, Abrasifs appliqués- Granulométrie. ISO 6344-1,2,3.

Uhlmanna et al. (2016) Uhlmanna, E., Koprowskia, S., Weingaertnerc, W.L., Rolon D.A., 2016. Modelling and simulation of grinding processes with mounted points: Part 
655 II of II - Fast modelling method for workpiece surface prediction. CIRP conference on high performance cutting. 46, 603-606.

Wang et al. (2009) Wang, G., Wang, Y., Xu, Z., 2009. Modeling and analysis of the material removal depth for stone polishing. Journal of materials processing technology. 209, 2453-2463.

660 Wu et al. (2020) Wu, J., Cheng, J., Gao, C., Yu, T., Guo Z., 2020. Research on predicting model of surface roughness in small-scale grinding of brittle materials considering grinding tool topography. International Journal of Mechanical Sciences. $166,105263$.

Xi and Zhou (2005) Xi, F., Zhou, D., 2005. Modeling surface roughness in the stone 665 polishing process. International Journal of Machine Tools \& Manufacture. 45, 365-372.

Zhang et al. (2020) Zhang, J., Wang, H., Senthil Kumar, A., Mingsheng Jin, M. 2020. Experimental and theoretical study of internal finishing by a novel magnetically driven polishing tool. International Journal of Machine Tools and Manufacture. 153, 103552 .

670 Zhao et al. (2014) Zhao, T., Shi, Y., Lin, X., Duan, J., Sun, P., Zhang, J., 2014. Surface roughness prediction and parameters optimization in grinding and polishing process for IBR of aero-engine. Int. J. Adv. Manuf. Technol. 74, 653-663.

Zhou and Xi (2002) Zhou, X., Xi, F., 2002. Modeling and predicting surface roughness of the grinding process. International Journal of Machine Tools \& 675 Manufacture. 42, 969-977. 